\title{
Erratum to: Long non-coding RNA linc00673 regulated non-small cell lung cancer proliferation, migration, invasion and epithelial mesenchymal transition by sponging miR-150-5p
}

Wei Lu ${ }^{1,2+}$, Honghe Zhang ${ }^{3+}$, Yuequn Niu ${ }^{1+}$, Yongfeng Wu${ }^{1}$, Wenjie Sun ${ }^{3}$, Hongyi $\mathrm{Li}^{1}$, Jianlu Kong ${ }^{3}$, Kefeng Ding ${ }^{2}$, Han-Ming Shen ${ }^{4}$, Han Wu $\mathrm{Wu}^{5}$, Dajing $\mathrm{Xia}^{1^{*}}$ and Yihua $\mathrm{Wu}^{1^{*}}$

\section{Erratum}

After the publication of this work [1] an error was noticed in Additional file 1: Table S1 regarding the siRNA sequences, in which two siRNAs (si-L4/L5) were omitted from the figure. The updated version (now correctly added) has the sequences of si-L1/L2/L3 and the other two, si-L4/L5. Since the last two siRNAs (si-L4/L5) served as further confirmation for the original data, this update does not affect the findings or conclusions of the article. Nevertheless, we apologize for the inconvenience.
Received: 22 August 2017 Accepted: 22 August 2017

Published online: 29 August 2017

\section{Reference}

1. Lu W, Zhang H, Niu Y, Wu Y, Sun W, Li H, et al. Long non-coding RNA linc00673 regulated non-small cell lung cancer proliferation, migration, invasion and epithelial mesenchymal transition by sponging miR-150-5p. Mol Cancer. 2017;16:118.

\section{Additional file}

Additional file 1: siRNA sequences. (DOCX $15 \mathrm{~kb})$

\section{Author details}

'Department of Toxicology, Zhejiang University School of Public Health, 866 Yuhangtang Road, Hangzhou, People's Republic of China. ${ }^{2}$ Department of Surgical Oncology, Second Affiliated Hospital, Zhejiang University School of Medicine, Hangzhou, People's Republic of China. ${ }^{3}$ Department of Pathology, Zhejiang University School of Medicine, Hangzhou, People's Republic of China. ${ }^{4}$ Department of Physiology, Yong Loo Lin School of Medicine, National University of Singapore, Singapore, Singapore. ${ }^{5}$ Department of Ophthalmology, Second Affiliated Hospital, Zhejiang University School of Medicine, Hangzhou, People's Republic of China.

\footnotetext{
*Correspondence: dxia@Zju.Edu.Cn; georgewuer@126.Com

${ }^{\dagger}$ Equal contributors

'Department of Toxicology, Zhejiang University School of Public Health, 866 Yuhangtang Road, Hangzhou, People's Republic of China
} 